\title{
Existence of positive solutions to elliptic problems involving the fractional Laplacian
}

Bin $\mathrm{Ge}^{1 *}$ and Chao Zhang ${ }^{2}$

\begin{abstract}
This paper focuses on the following elliptic problem involving a fractional Laplacian:

$$
(-\Delta)^{\alpha} u+V(x) u=f(u) \text { in } \mathbb{R}^{N}
$$

where $N \geq 2, \alpha \in(0,1),(-\Delta)^{\alpha}$ stands for the fractional Laplacian. Using some variational methods, we obtain the existence of positive solutions without compactness conditions.
\end{abstract}

MSC: $34 \mathrm{~A} 08 ; 35 \mathrm{~A} 15 ; 58 \mathrm{E} 30$

Keywords: fractional-Laplacian; variational method; positive solutions; Pohozaev type identity

\section{Introduction}

In this paper, we consider the existence of positive solutions to the following fractional Schrödinger equation:

$$
(-\Delta)^{\alpha} u+V(x) u=f(u) \text { in } \mathbb{R}^{N}
$$

where $N \geq 2, \alpha \in(0,1),(-\Delta)^{\alpha}$ stands for the fractional Laplacian, $f \in C\left(\mathbb{R}_{+}, \mathbb{R}_{+}\right)$. The fractional Laplacian $(-\Delta)^{\alpha}$ with $\alpha \in(0,1)$ of a function $\phi \in \mathcal{S}$ is defined by

$$
\mathcal{F}\left(\left((-\Delta)^{\alpha}\right) \phi\right)(\xi)=|\xi|^{2 \alpha} \mathcal{F}(\phi)(\xi), \quad \forall \alpha \in(0,1)
$$

where $\mathcal{S}$ denotes the Schwartz space of rapidly decreasing $C^{\infty}$ functions in $\mathbb{R}^{N}, \mathcal{F}$ is the Fourier transform, i.e.,

$$
\mathcal{F}(\phi)(\xi)=\frac{1}{(2 \pi)^{N / 2}} \int_{\mathbb{R}^{N}} e^{-2 \pi i \xi \cdot x} \phi(x) d x
$$

In the past few years, there have been research activities (see [1-6], etc.) on the study of existence and multiplicity of solutions for such kind of problems (P). Here we just mention some results related to our problems.

- For the case $V \equiv 1$, the authors in [5] studied the existence of positive solutions of (P) when $f$ has subcritical growth and satisfies the Ambrosetti-Rabinowitz condition. 
- For the case $V \equiv 0$, Chang and Wang in [3] obtained the existence of a positive ground state under the general Berestycki-Lions type assumptions.

- Moreover, for the general potential $V$, which is allowed to vary, ground states were found by imposing a coercivity assumption on $V$, i.e.,

$$
\lim _{|x| \rightarrow+\infty} V(x)=+\infty
$$

We refer the readers to $[4,6]$ for details. Recently, when the potential $V$ satisfies the conditions $H(V)(1)$ and $H(V)(3)$, Chang in [2] proved the existence of ground state solutions under the assumption that $f(u)$ is asymptotically linear with respect to $u$.

Inspired by the above-mentioned papers, we are concerned with the existence of positive solutions of $(\mathrm{P})$. The novelties in this paper are mainly two parts. First, we just assume that the nonlinear term $f(u)$ is superlinear with respect to $u$ at infinity instead of the asymptotically linear condition or Ambrosetti-Rabinowitz condition, which is completely different from those appearing in the literature. To compensate the lack of compactness, we employ the Pohozaev identity to obtain the boundedness of Palais-Smale sequence. Second, it is worth mentioning that in the present paper we consider the existence of non-radial positive solutions of $(\mathrm{P})$. We have to prove a new compact embedding theorem by virtue of some assumptions imposed on the potential $V$.

Throughout this paper, we assume:

$H(V): \quad(1) \quad V \in C^{1}\left(\mathbb{R}^{N}, \mathbb{R}^{N}\right), V_{0}:=\inf _{x \in \mathbb{R}^{N}} V(x)>0$.

(2) $\langle\nabla V(x), x\rangle \in L^{\frac{N}{2 \alpha}}\left(\mathbb{R}^{N}\right)$ and

$$
|\langle\nabla V(x), x\rangle|_{L^{\frac{N}{2 \alpha}\left(\mathbb{R}^{N}\right)}}<2 \alpha S_{\alpha},
$$

where $S_{\alpha}$ is the best Sobolev constant of the embedding

$$
\dot{H}^{\alpha}\left(\mathbb{R}^{N}\right) \hookrightarrow L^{2_{\alpha}^{*}}\left(\mathbb{R}^{N}\right) \text {, i.e., } S_{\alpha}=\inf _{u \in \dot{H}^{\alpha}\left(\mathbb{R}^{N}\right)} \frac{\int_{\mathbb{R}^{N}}\left|(-\Delta)^{\frac{\alpha}{2}} u\right|^{2} d x}{|u|_{2_{\alpha}^{*}}^{*}} \text { (see [7]). }
$$

(3) There exists $r>0$ such that, for any $b>0$,

$$
\lim _{|y| \rightarrow \infty} \mu\left(\left\{x \in \mathbb{R}^{N}: V(x) \leq b\right\} \cap B_{r}(y)\right)=0,
$$

where $\mu$ is the Lebesgue measure on $\mathbb{R}^{N}$.

$H(f): \quad$ (1) $f: \mathbb{R}_{+} \rightarrow \mathbb{R}_{+}$is of class $C^{1, \gamma}$ for some $\gamma>\max \{0,1-2 \alpha\}$.

(2) $|f(u)| \leq C\left(|u|+|u|^{p-1}\right)$ for all $u \in \mathbb{R}_{+}=[0,+\infty)$ and $p \in\left(2,2_{\alpha}^{*}\right)$, where $2_{\alpha}^{*}=\frac{2 N}{N-2 \alpha}$ for $N \geq 2$.

(3) $\lim _{u \rightarrow 0} \frac{f(u)}{u}=0$.

(4) $\lim _{u \rightarrow \infty} \frac{f(u)}{u}=\infty$.

Next, we state our main result.

Theorem 1 Assume that $H(V)$ and $H(f)$ hold. Then problem (P) has at least one positive solution.

The rest of this paper is organized as follows. In Section 2, we state and prove some preliminary results which will be used later. We will finish the proof of our main result (Theorem 1) in Section 3. 


\section{Preliminary}

In this section we recall some results on Sobolev spaces of fractional order. A very complete introduction to fractional Sobolev spaces can be found in [8].

Consider the fractional order Sobolev space

$$
H^{\alpha}\left(\mathbb{R}^{N}\right)=\left\{u \in L^{2}\left(\mathbb{R}^{N}\right): \int_{\mathbb{R}^{N}}\left(|\xi|^{2 \alpha} \hat{u}^{2}+\hat{u}^{2}\right) d \xi<+\infty\right\}
$$

where $\hat{u} \doteq \mathcal{F}(u)$. The norm is defined by

$$
\|u\|_{H^{\alpha}\left(\mathbb{R}^{N}\right)}=\left(\int_{\mathbb{R}^{N}}\left(|\xi|^{2 \alpha} \hat{u}^{2}+\hat{u}^{2}\right) d \xi\right)^{\frac{1}{2}} .
$$

The homogeneous fractional Sobolev space $D^{\alpha, 2}\left(\mathbb{R}^{N}\right)$, also denoted by $\dot{H}^{\alpha}\left(\mathbb{R}^{N}\right)$, is defined as the completion of $C_{0}^{\infty}\left(\mathbb{R}^{N}\right)$ with respect to the norm

$$
\|u\|_{\dot{H}^{\alpha}\left(\mathbb{R}^{N}\right)}=\left(\int_{\mathbb{R}^{N}}|\xi|^{2 \alpha} \hat{u}^{2} d \xi\right)^{\frac{1}{2}}=\left(\int_{\mathbb{R}^{N}}\left|(-\Delta)^{\frac{\alpha}{2}} u(x)\right|^{2} d x\right)^{\frac{1}{2}} .
$$

In this paper we consider its subspace:

$$
E=\left\{u \in \dot{H}^{\alpha}\left(\mathbb{R}^{N}\right): \int_{\mathbb{R}^{N}} V(x) u^{2} d x<+\infty\right\}
$$

with the norm

$$
\|u\|_{E}=\left(\int_{\mathbb{R}^{N}}\left|(-\Delta)^{\frac{\alpha}{2}} u(x)\right|^{2} d x+\int_{\mathbb{R}^{N}} V(x) u^{2} d x\right)^{\frac{1}{2}} .
$$

Lemma $1([5]) H^{\alpha}\left(\mathbb{R}^{N}\right)$ continuously embedded into $L^{p}\left(\mathbb{R}^{N}\right)$ for $p \in\left[2,2_{\alpha}^{*}\right]$, and compactly embedded into $L_{\mathrm{loc}}^{p}\left(\mathbb{R}^{N}\right)$ for $p \in\left[2,2_{\alpha}^{*}\right)$.

Using the above lemma, we can obtain the following result.

Theorem 2 Assume that $H(V)(1)$ and $H(V)(3)$ hold. Then:

(i) we have a compact embedding $E \hookrightarrow L^{2}\left(\mathbb{R}^{N}\right)$;

(ii) for any $p \in\left(2,2_{\alpha}^{*}\right)$, we have a compact embedding $E \hookrightarrow L^{p}\left(\mathbb{R}^{N}\right)$.

Proof (i) Assume $u_{n} \rightarrow 0$ in $E$, and $\left\|u_{n}\right\|_{E} \leq c_{1}$. We need to show $u_{n} \rightarrow 0$ in $L^{2}\left(\mathbb{R}^{N}\right)$. By Lemma 1 , we have $u_{n} \rightarrow 0$ in $L_{\text {loc }}^{2}\left(\mathbb{R}^{N}\right)$. It suffices to show that for every $\varepsilon>0$, there exists $R>0$ such that

$$
\int_{B_{R}^{c}}\left|u_{n}\right|^{2} d x \leq \varepsilon \quad \text { for all } n=1,2, \ldots
$$

where $B_{R}=\left\{x \in \mathbb{R}^{N}:|x|<R\right\}, B_{R}^{c}=\left\{x \in \mathbb{R}^{N}:|x| \geq R\right\}$. 
Ge and Chang Boundary Value Problems (2015) 2015:235

Page 4 of 12

First of all, choose $\left\{y_{i}\right\} \subset \mathbb{R}^{N}$ such that $\mathbb{R}^{N} \subset \bigcup_{i=1}^{\infty} B_{r}\left(y_{i}\right)$ and each $x \in \mathbb{R}^{N}$ is covered by at most $2^{N}$ such balls. So

$$
\begin{aligned}
\int_{B_{R}^{c}}\left|u_{n}\right|^{2} d x & \leq \sum_{\left|y_{i}\right| \geq R-r} \int_{B_{r}\left(y_{i}\right)}\left|u_{n}\right|^{2} d x \\
& =\sum_{\left|y_{i}\right| \geq R-r}\left[\int_{B_{r}\left(y_{i}\right) \cap\left\{x \in \mathbb{R}^{N}: V(x)>b\right\}}\left|u_{n}\right|^{2} d x+\int_{A_{b}\left(y_{i}\right)}\left|u_{n}\right|^{2} d x\right],
\end{aligned}
$$

where $A_{b}\left(y_{i}\right)=B_{r}\left(y_{i}\right) \cap\left\{x \in \mathbb{R}^{N}: V(x) \leq b\right\}$.

On the one hand,

$$
\int_{B_{r}\left(y_{i}\right) \cap\left\{x \in \mathbb{R}^{N}: V(x)>b\right\}}\left|u_{n}\right|^{2} d x \leq \frac{1}{b} \int_{B_{r}\left(y_{i}\right)} V(x)\left|u_{n}\right|^{2} d x,
$$

where $B_{r}(y)=\left\{x \in \mathbb{R}^{N}:|x-y|<r\right\}$.

On the other hand, since Sobolev embedding $E \hookrightarrow H^{\alpha}\left(\mathbb{R}^{N}\right) \hookrightarrow L^{2_{\alpha}^{*}}\left(\mathbb{R}^{N}\right)$ is continuous, there is a constant $c_{2}>0$ such that

$$
\left|u_{n}\right|_{2_{\alpha}^{*}} \leq c_{2}\left\|u_{n}\right\|_{E} \leq c_{1} c_{2}
$$

Applying the Hölder inequality, we get

$$
\begin{aligned}
\int_{A_{b}\left(y_{i}\right)}\left|u_{n}\right|^{2} d x & \leq\left(\int_{A_{b}\left(y_{i}\right)}\left|u_{n}\right|^{\frac{2 N}{N-2 \alpha}} d x\right)^{\frac{N-2 \alpha}{N}}\left(\int_{A_{b}\left(y_{i}\right)} 1^{\frac{N}{2 \alpha}} d x\right)^{\frac{2 \alpha}{N}} \\
& \leq\left(\int_{A_{b}\left(y_{i}\right)}\left|u_{n}\right|^{\frac{2 N}{N-2 \alpha}} d x\right)^{\frac{N-2 \alpha}{N}} \sup _{\left|y_{i}\right| \geq R-r}\left[\mu\left(A_{b}\left(y_{i}\right)\right)\right]^{\frac{2 \alpha}{N}} .
\end{aligned}
$$

Hence,

$$
\begin{aligned}
\int_{B_{R}^{c}}\left|u_{n}\right|^{2} d x \leq & \sum_{\left|y_{i}\right| \geq R-r}\left[\frac{1}{b} \int_{B_{r}\left(y_{i}\right)} V(x)\left|u_{n}\right|^{2} d x\right. \\
& \left.+\left(\int_{A_{b}\left(y_{i}\right)}\left|u_{n}\right|^{\frac{2 N}{N-2 \alpha}} d x\right)^{\frac{N-2 \alpha}{N}} \sup _{\left|y_{i}\right| \geq R-r}\left[\mu\left(A_{b}\left(y_{i}\right)\right)\right]^{\frac{2 \alpha}{N}}\right] \\
\leq & \frac{2^{N}}{b} \int_{B_{R-2 r}^{c}} V(x)\left|u_{n}\right|^{2} d x \\
& +\left(2^{N} \int_{B_{R-2 r}^{c}}\left|u_{n}\right|^{\frac{2 N}{N-2 \alpha}} d x\right)^{\frac{N-2 \alpha}{N}} \sup _{\left|y_{i}\right| \geq R-r}\left[\mu\left(A_{b}\left(y_{i}\right)\right)\right]^{\frac{2 \alpha}{N}} \\
\leq & \frac{2^{N}}{b} \int_{\mathbb{R}^{N}} V(x)\left|u_{n}\right|^{2} d x \\
& +2^{N-2 \alpha}\left(\int_{\mathbb{R}^{N}}\left|u_{n}\right|^{\frac{2 N}{N-2 \alpha}} d x\right)^{\frac{N-2 \alpha}{N}} \sup _{|y| \geq R-r}\left[\mu\left(A_{b}(y)\right)\right]^{\frac{2 \alpha}{N}} \\
\leq & \frac{2^{N}}{b} \int_{\mathbb{R}^{N}}\left(\left|(-\Delta)^{\frac{\alpha}{2}} u_{n}\right|^{2}+V(x)\left|u_{n}\right|^{2}\right) d x
\end{aligned}
$$




$$
\begin{aligned}
& +2^{N-2 \alpha}\left|u_{n}\right|_{2_{\alpha}^{*}}^{2} \sup _{|y| \geq R-r}\left[\mu\left(A_{b}(y)\right)\right]^{\frac{2 \alpha}{N}} \\
\leq & \frac{2^{N}}{b}\left\|u_{n}\right\|_{E}^{2}+2^{N-2 \alpha} c_{2}^{2}\left\|u_{n}\right\|_{E}^{2} \sup _{|y| \geq R-r}\left[\mu\left(A_{b}(y)\right)\right]^{\frac{2 \alpha}{N}} \\
\leq & \frac{2^{N}}{b} c_{1}^{2}+2^{N-2 \alpha} c_{2}^{2} c_{1}^{2} \sup _{|y| \geq R-r}\left[\mu\left(A_{b}(y)\right)\right]^{\frac{2 \alpha}{N}} .
\end{aligned}
$$

Now, choose $b>0$ such that

$$
\frac{2^{N}}{b} c_{1}^{2}<\frac{\varepsilon}{2} \text {. }
$$

For such a fixed $b>0$, since

$$
\sup _{|y| \geq R-r}\left[\mu\left(A_{b}(y)\right)\right]^{\frac{2 \alpha}{N}} \rightarrow 0 \quad \text { as } R \rightarrow+\infty,
$$

there exists $R>0$ large enough such that

$$
\sup _{|y| \geq R-r}\left[\mu\left(A_{b}(y)\right)\right]^{\frac{2 \alpha}{N}} \leq \frac{\varepsilon}{2^{N-2 \alpha+1} c_{2}^{2} c_{1}^{2}} .
$$

It follows from (1) and (2) that

$$
\int_{B_{R}^{c}}\left|u_{n}\right|^{2} d x \leq \varepsilon
$$

from which conclusion (i) of the lemma follows.

(ii) To prove the lemma for general exponent $p \in\left(2,2_{\alpha}^{*}\right)$, we use an interpolation argument. Let $u_{n} \rightarrow 0$ in $E$, we have just proved that $u_{n} \rightarrow 0$ in $L^{2}\left(\mathbb{R}^{N}\right)$.

Moreover, since the embedding $E \hookrightarrow L^{2_{\alpha}^{*}}\left(\mathbb{R}^{N}\right)$ is continuous and $\left\{u_{n}\right\}$ is bounded in $E$, we also have $\sup _{n} \int_{\mathbb{R}^{N}}\left|u_{n}\right|^{2 *} d x<+\infty$.

Since $2<p<2_{\alpha}^{*}$, there exists $\lambda \in(0,1)$ such that

$$
\frac{1}{p}=\frac{\lambda}{2}+\frac{1-\lambda}{2_{\alpha}^{*}}
$$

Then we have

$$
s=\frac{2}{p \lambda}>1 \quad \text { and } \quad t=\frac{2_{\alpha}^{*}}{p(1-\lambda)}>1 .
$$

Using Hölder's inequality, we deduce that

$$
\begin{aligned}
\int_{\mathbb{R}^{N}}\left|u_{n}\right|^{p} d x & =\int_{\mathbb{R}^{N}}\left|u_{n}\right|^{\frac{2}{s}}\left|u_{n}\right|^{\frac{2 *}{t}} d x \\
& \leq\left.\left.\left.\left.|| u_{n}\right|^{\frac{2}{s}}\right|_{s}|| u_{n}\right|^{\frac{2_{\alpha}^{*}}{t}}\right|_{t} \\
& =\left|u_{n}\right|_{2}^{\frac{2}{s}}\left|u_{n}\right|_{2_{\alpha}^{*}}^{\frac{2_{\alpha}^{*}}{t}} \rightarrow 0 \quad \text { as } n \rightarrow+\infty .
\end{aligned}
$$

This implies $u_{n} \rightarrow 0$ in $L^{p}\left(\mathbb{R}^{N}\right)$, and the proof of conclusion (ii) is completed. 
Next, we state the following version of the Pohozaev identity, which will be used to obtain the boundedness of $\left\|u_{n}\right\|_{E}$. Similar results can be found in [3, 8-10]. Its proof is a mixture of many ingredients that are scattered through the literature. We refer the readers to Proposition 4.1 in [10] (see also Proposition 4.1 in [3] for the case that $V(x) \equiv 0$ ) for the details.

Lemma 2 Let $N \geq 2$. Assume that $H(f)(1)$ and $H(f)(2)$ hold. If $u \in E$ is a weak solution of (P), then the following Pohozaev type identity holds:

$$
\begin{aligned}
& \frac{N-2 \alpha}{2} \int_{\mathbb{R}^{N}}\left|(-\Delta)^{\frac{\alpha}{2}} u\right|^{2} d x+\frac{N}{2} \int_{\mathbb{R}^{N}} V(x)|u|^{2} d x+\frac{1}{2} \int_{\mathbb{R}^{N}}\langle\nabla V(x), x\rangle|u(x)|^{2} d x \\
& =N \int_{\mathbb{R}^{N}} F(u(x)) d x .
\end{aligned}
$$

Remark 1 We would like to mention that the regularity condition $H(f)(1)$ is necessary to prove the Pohozaev identity. The smoothness of $f$ will only be used here.

In order to discuss the problem (P), we need to define a functional in $E$ :

$$
\varphi(u)=\frac{1}{2} \int_{\mathbb{R}^{N}}\left(\left|(-\Delta)^{\frac{\alpha}{2}} u(x)\right|^{2}+V(x)|u(x)|^{2}\right) d x-\int_{\mathbb{R}^{N}} F(u(x)) d x, \quad \forall u \in E .
$$

Then we see from $H(f)(1)$ and $H(f)(2)$ that $\varphi$ is well defined on $E$ and is of $C^{1}$, and

$$
\left\langle\varphi^{\prime}(u), v\right\rangle=\int_{\mathbb{R}^{N}}\left((-\Delta)^{\frac{\alpha}{2}} u(-\Delta)^{\frac{\alpha}{2}} v+V(x) u v\right) d x-\int_{\mathbb{R}^{N}} f(u) v d x, \quad \forall u, v \in E .
$$

It is standard to verify that the weak solutions of $(\mathrm{P})$ correspond to the critical points of the functional $\varphi$.

Next we recall a monotonicity method due to Struwe [11] and Jeanjean [12], which will be used in our proof. The version here is from [12].

Theorem 3 Let $(X,\|\cdot\|)$ be a Banach space and $I \subset \mathbb{R}_{+}$an interval. Consider the family of $C^{1}$ functionals on $X$,

$$
\varphi_{\lambda}(u)=A(u)-\lambda B(u), \quad \lambda \in I,
$$

with $B$ nonnegative and either $A(u) \rightarrow \infty$ or $B(u) \rightarrow \infty$ as $\|u\| \rightarrow \infty$ and such that $\varphi_{\lambda}(0)=0$.

For any $\lambda \in I$ we set $\mathcal{T}_{\lambda}=\left\{\gamma \in C([0,1], X): \gamma(0)=0, \varphi_{\lambda}(\gamma(1))<0\right\}$.

If for every $\lambda \in I$ the set $\mathcal{T}_{\lambda}$ is nonempty and

$$
c_{\lambda}=\inf _{\gamma \in \mathcal{T}_{\lambda}} \max _{t \in[0,1]} \varphi_{\lambda}(\gamma(t))>0
$$

then for almost every $\lambda \in I$ there is a sequence $\left\{u_{n}\right\} \subset X$ such that:

(1) $\left\{u_{n}\right\}$ is bounded;

(2) $\varphi_{\lambda}\left(u_{n}\right) \rightarrow c_{\lambda}$ as $n \rightarrow \infty$;

(3) $\varphi_{\lambda}^{\prime}\left(u_{n}\right) \rightarrow 0$ as $n \rightarrow \infty$, in the dual space $X^{-1}$ of $X$. 
In our case, $X=E$,

$$
A(u)=\frac{1}{2} \int_{\mathbb{R}^{N}}\left(\left|(-\Delta)^{\frac{\alpha}{2}} u\right|^{2}+V(x) u^{2}\right) d x \quad \text { and } \quad B(u)=\int_{\mathbb{R}^{N}} F(u) d x .
$$

\section{Some lemmas and proof of main result}

In this section, to overcome the lack of compactness, we need to consider the functional $\varphi_{\lambda}$ in the functions space $E$. We shall prove that $\varphi_{\lambda}$ satisfies the conditions of Theorem 3 in the next several lemmas.

Lemma 3 Let $\mathcal{T}_{\lambda}$ be the set of paths defined in Theorem 3. Then $\mathcal{T}_{\lambda} \neq \emptyset$ for $\lambda \in I=[\delta, 1]$, where $\delta \in(0,1)$ is a positive constant.

Proof We choose $\eta \in C_{0}^{\infty}\left(\mathbb{R}^{N}\right)$ with $\|\eta\|_{E}=1$ and $\sup \eta \subset B_{r}(0)$ for some $r>0$. From $H(f)(4)$, we know that for any $c_{3}>0$ with $c_{3} \delta \int_{B_{r}(0)} \eta^{2} d x>1 / 2$, there exists $c_{4}>0$ such that

$$
F(u) \geq c_{3}|u|^{2}-c_{4}, \quad u \in \mathbb{R}_{+}
$$

Then we have

$$
\begin{aligned}
\varphi_{\lambda}(t \eta) & =\frac{t^{2}}{2} \int_{\mathbb{R}^{N}}\left(\left|(-\Delta)^{\frac{\alpha}{2}} \eta\right|^{2}+V(x) \eta^{2}\right) d x-\lambda \int_{\mathbb{R}^{N}} F(t \eta) d x \\
& =\frac{t^{2}}{2}-\lambda \int_{\mathbb{R}^{N}} F(t \eta) d x \\
& \leq \frac{t^{2}}{2}-\delta c_{3} t^{2} \int_{B_{r}(0)} \eta^{2} d x+c_{4}\left|B_{r}(0)\right| \\
& =t^{2}\left(\frac{1}{2}-\delta c_{3} \int_{B_{r}(0)} \eta^{2} d x\right)+c_{4}\left|B_{r}(0)\right|
\end{aligned}
$$

Therefore, we can choose $t>0$ large such that $\varphi_{\lambda}(t \eta)<0$. The proof is completed.

Lemma 4 Let $c_{\lambda}$ be the set of paths defined in Theorem 3 . Then there exists a constant $\bar{c}>0$ such that $c_{\lambda} \geq \bar{c}$ for $\lambda \in[\delta, 1]$.

Proof By $H(f)(2)$ and $H(f)(3)$, for any $\varepsilon \in(0,1 / 2)$, there exists $c_{\varepsilon}>0$ such that

$$
|F(u)| \leq \frac{\varepsilon V_{0}}{2}|u|^{2}+c_{\varepsilon}|u|^{p}, \quad u \in \mathbb{R}_{+} .
$$

Furthermore, by Theorem 2, we have $E \hookrightarrow L^{p}\left(\mathbb{R}^{N}\right)$ compactly. Then there exists $c_{5}>0$ such that $|u|_{p} \leq c_{5}\|u\|_{E}$. Hence, for any $u \in E$ and $\lambda \in[\delta, 1]$, using (4) it follows that

$$
\begin{aligned}
\varphi_{\lambda}(u) & =\frac{1}{2} \int_{\mathbb{R}^{N}}\left(\left|(-\Delta)^{\frac{\alpha}{2}} u\right|^{2}+V(x) u^{2}\right) d x-\lambda \int_{\mathbb{R}^{N}} F(u) d x \\
& \geq \frac{1}{2}\|u\|_{E}^{2}-\int_{\mathbb{R}^{N}}\left(\frac{\varepsilon V_{0}}{2}|u|^{2}+c_{\varepsilon}|u|^{p}\right) d x \\
& \geq \frac{1}{2}\|u\|_{E}^{2}-\frac{\varepsilon}{2} \int_{\mathbb{R}^{N}} V(x)|u|^{2} d x-c_{\varepsilon} \int_{\mathbb{R}^{N}}|u|^{p} d x
\end{aligned}
$$




$$
\begin{aligned}
& \geq \frac{1}{2}\|u\|_{E}^{2}-\frac{\varepsilon}{2} \int_{\mathbb{R}^{N}}\left(\left|(-\Delta)^{\frac{\alpha}{2}} u\right|^{2}+V(x)|u|^{2}\right) d x-c_{\varepsilon} \int_{\mathbb{R}^{N}}|u|^{p} d x \\
& \geq \frac{1}{4}\|u\|_{E}^{2}-c_{\varepsilon}|u|_{p}^{p} \\
& \geq \frac{1}{4}\|u\|_{E}^{2}-c_{\varepsilon} c_{5}^{p}\|u\|_{E}^{p}
\end{aligned}
$$

which implies that there exists $\rho>0$ such that $\varphi_{\lambda}(u)>0$ for every $u \in E$ and $\|u\|_{E} \in(0, \rho]$. In particular, for $\|u\|_{E}=\rho$, we have $\varphi_{\lambda}(u) \geq \bar{c}>0$. Fix $\lambda \in[\delta, 1]$ and $\gamma \in \mathcal{T}_{\lambda}$. By the definition of $\mathcal{T}_{\lambda}$ we can see that $\|\gamma(1)\|_{E}>\rho$. By continuity, we deduce that there exists $t_{\gamma} \in(0,1)$ such that $\left\|\gamma\left(t_{\gamma}\right)\right\|_{E}=\rho$. Thus, for any $\lambda \in[\delta, 1]$,

$$
c_{\lambda} \geq \inf _{\gamma \in \mathcal{T}_{\lambda}} \varphi_{\lambda}\left(\gamma\left(t_{\gamma}\right)\right) \geq \bar{c}>0
$$

Therefore, we complete the proof.

Next we prove that the functional $\varphi_{\lambda}$ can achieve the critical value at $c_{\lambda}$ for any $\lambda \in[\delta, 1]$.

Lemma 5 For any $\lambda \in[\delta, 1]$, each bounded (PS) sequence of the functional $\varphi_{\lambda}$ admits a convergent subsequence.

Proof Let $\lambda \in[\delta, 1]$. Suppose that $\left\{u_{n}\right\} \subset E$ is a $(P S)$ sequence for $\varphi_{\lambda}$, that is, $\left\{u_{n}\right\}$ and $\varphi_{\lambda}\left(u_{n}\right)$ are bounded, $\varphi_{\lambda}^{\prime}\left(u_{n}\right) \rightarrow 0$ in $E^{\prime}$, where $E^{\prime}$ is the dual space of $E$. Then there exists $u \in E$ such that $u_{n} \rightarrow u$ in $E$. Thus, Theorem 2 implies that

$$
\begin{array}{ll}
u_{n} \rightarrow u & \text { in } L^{p}\left(\mathbb{R}^{N}\right), \\
u_{n} \rightarrow u & \text { a.e. in } \mathbb{R}^{N} .
\end{array}
$$

By virtue of hypothesis $H(f)(2)$ and $H(f)(3)$, for any $\varepsilon \in(0,1 / 2)$, there exists $c_{\varepsilon}>0$ such that

$$
|f(u)| \leq \varepsilon|u|+c_{\varepsilon}|u|^{p-1}, \quad u \in \mathbb{R}_{+} .
$$

So it follows from (5) that

$$
\begin{aligned}
\left|\int_{\mathbb{R}^{N}} f\left(u_{n}\right)\left(u_{n}-u\right) d x\right| & \leq \int_{\mathbb{R}^{N}}\left|f\left(u_{n}\right)\right|\left|u_{n}-u\right| d x \\
& \leq \int_{\mathbb{R}^{N}}\left(\varepsilon\left|u_{n}\right|+c_{\varepsilon}\left|u_{n}\right|^{p-1}\right)\left|u_{n}-u\right| d x \\
& \leq \varepsilon\left|u_{n}\right|_{2}\left|u_{n}-u\right|_{2}+\left.\left.c_{\varepsilon}|| u_{n}\right|^{p-1}\right|_{p^{\prime}}\left|u_{n}-u\right|_{p} \\
& \leq \varepsilon\left|u_{n}\right|_{2}\left|u_{n}-u\right|_{2}+c_{\varepsilon}\left|u_{n}\right|_{p}^{p-1}\left|u_{n}-u\right|_{p} \\
& \leq \varepsilon c_{5}\left\|u_{n}\right\|_{E}\left|u_{n}-u\right|_{2}+c_{\varepsilon} c_{5}^{p-1}\left\|u_{n}\right\|_{E}^{p-1}\left|u_{n}-u\right|_{p}
\end{aligned}
$$

which implies that

$$
\int_{\mathbb{R}^{N}} f\left(u_{n}\right)\left(u_{n}-u\right) d x \rightarrow 0 \quad \text { as } n \rightarrow \infty .
$$


Thus

$$
\begin{aligned}
& \left\langle\varphi_{\lambda}^{\prime}\left(u_{n}\right), u_{n}-u\right\rangle \\
& =\int_{\mathbb{R}^{N}}\left((-\Delta)^{\frac{\alpha}{2}} u_{n}(-\Delta)^{\frac{\alpha}{2}}\left(u_{n}-u\right)+V(x) u_{n}\left(u_{n}-u\right)\right) d x \\
& \quad-\lambda \int_{\mathbb{R}^{N}} f\left(u_{n}\right)\left(u_{n}-u\right) d x \\
& =\int_{\mathbb{R}^{N}}\left((-\Delta)^{\frac{\alpha}{2}} u_{n}(-\Delta)^{\frac{\alpha}{2}}\left(u_{n}-u\right)+V(x) u_{n}\left(u_{n}-u\right)\right) d x+o(1)
\end{aligned}
$$

and

$$
\int_{\mathbb{R}^{N}}\left((-\Delta)^{\frac{\alpha}{2}} u_{n}(-\Delta)^{\frac{\alpha}{2}}\left(u_{n}-u\right)+V(x) u_{n}\left(u_{n}-u\right)\right) d x \rightarrow 0 \quad \text { as } n \rightarrow \infty
$$

Therefore we conclude that $\left\|u_{n}\right\|_{E}^{2} \rightarrow\|u\|_{E}^{2}$. This together with $u_{n} \rightarrow u$ in $E$ shows that $u_{n} \rightarrow u$ in $E$. The proof is completed.

Now we are in the position to show that the modified functional $\varphi_{\lambda}$ has a nontrivial critical point.

Lemma 6 For almost every $\lambda \in[\delta, 1]$, there exists $u_{\lambda} \in E \backslash\{0\}$ such that $\varphi_{\lambda}^{\prime}\left(u_{\lambda}\right)=0$ and $\varphi_{\lambda}\left(u_{\lambda}\right)=c_{\lambda}$.

Proof By virtue of Theorem 3, for almost every $\lambda \in[\delta, 1]$, there exists a bounded sequence $\left\{u_{\lambda}^{n}\right\} \subset E$ such that

$$
\varphi_{\lambda}\left(u_{\lambda}^{n}\right) \rightarrow c_{\lambda} \quad \text { and } \quad \varphi_{\lambda}^{\prime}\left(u_{\lambda}^{n}\right) \rightarrow 0 \quad \text { as } n \rightarrow \infty
$$

According to Lemma 5, we may assume that there exists $u_{\lambda} \in E$ such that $u_{\lambda}^{n} \rightarrow u_{\lambda}$ in $E$. Then it follows that $\varphi_{\lambda}\left(u_{\lambda}\right)=c_{\lambda}$ and $\varphi_{\lambda}^{\prime}\left(u_{\lambda}\right)=0$ and $u_{\lambda} \neq 0$ from Lemma 4 .

From Lemma 6 we know that there exist a sequence $\lambda_{n} \in[\delta, 1]$ with $\lambda_{n} \rightarrow 1^{-}$and an associated sequence $\left\{u_{n}\right\} \subset E$ such that

$$
\varphi_{\lambda_{n}}\left(u_{n}\right)=c_{\lambda_{n}} \text { and } \quad \varphi_{\lambda_{n}}^{\prime}\left(u_{n}\right)=0
$$

Next, we will show that the sequence $\left\{u_{n}\right\}$ is bounded, which is a key ingredient in this paper.

Lemma 7 Let $u_{n}$ be a critical point of $\varphi_{\lambda_{n}}$ at the level $c_{\lambda_{n}}$ as defined in (6). Then there exists a constant $c_{6}>0$ such that $\left\|u_{n}\right\|_{E} \leq c_{6}$ for all $n$.

Proof In view of Lemma 2 and (6), we see that $u_{n}$ satisfies the following Pohozaev identity:

$$
\begin{aligned}
& \frac{N-2 \alpha}{2} \int_{\mathbb{R}^{N}}\left|(-\Delta)^{\frac{\alpha}{2}} u_{n}\right|^{2} d x+\frac{N}{2} \int_{\mathbb{R}^{N}} V(x)\left|u_{n}\right|^{2} d x+\frac{1}{2} \int_{\mathbb{R}^{N}}\langle\nabla V(x), x\rangle\left|u_{n}\right|^{2} d x \\
& =\lambda_{n} N \int_{\mathbb{R}^{N}} F\left(u_{n}\right) d x .
\end{aligned}
$$


Recall that $\varphi_{\lambda_{n}}\left(u_{n}\right)=c_{\lambda_{n}}$. So we have

$$
\frac{N}{2} \int_{\mathbb{R}^{N}}\left(\left|(-\Delta)^{\frac{\alpha}{2}} u_{n}\right|^{2}+V(x) u_{n}^{2}\right) d x-\lambda_{n} N \int_{\mathbb{R}^{N}} F\left(u_{n}\right) d x=c_{\lambda_{n}} N .
$$

Then from (7), (8), and hypothesis $H(f)(2)$, it follows that

$$
\begin{aligned}
\alpha \int_{\mathbb{R}^{N}}\left|(-\Delta)^{\frac{\alpha}{2}} u_{n}\right|^{2} d x & =c_{\lambda_{n}} N+\frac{1}{2} \int_{\mathbb{R}^{N}}\left\langle\nabla V(x),\left.x|| u_{n}\right|^{2} d x\right. \\
& \leq c_{\lambda_{n}} N+\frac{1}{2}|\langle\nabla V(x), x)|_{L^{\frac{N}{2 \alpha}\left(\mathbb{R}^{N}\right)}}\left|u_{n}\right|_{2_{\alpha}^{*}}^{2} \\
& \leq c_{\lambda_{n}} N+\frac{1}{2 S_{\alpha}}|\langle\nabla V(x), x)|_{L^{\frac{N}{2 \alpha}}\left(\mathbb{R}^{N}\right)} \int_{\mathbb{R}^{N}}\left|(-\Delta)^{\frac{\alpha}{2}} u_{n}\right|^{2} d x .
\end{aligned}
$$

Set $M_{0}=\alpha-\frac{1}{2 S_{\alpha}}|\langle\nabla V(x), x\rangle|_{L^{\frac{N}{2 \alpha}}\left(\mathbb{R}^{N}\right)}$. Then

$$
M_{0} \int_{\mathbb{R}^{N}}\left|(-\Delta)^{\frac{\alpha}{2}} u_{n}\right|^{2} d x \leq c_{\lambda_{n}} N
$$

We estimate the right-hand side of (9). By the min-max definition of the mountain pass level $c_{\lambda_{n}}$, Lemma 3 and (3), we have

$$
\begin{aligned}
c_{\lambda_{n}} & \leq \max _{t} \varphi_{\lambda_{n}}(t \eta) \\
& \leq \max _{t}\left\{\frac{t^{2}}{2}-\lambda_{n} \int_{\mathbb{R}^{N}} F(t \eta) d x\right\} \\
& \leq \max _{t}\left\{t^{2}\left(\frac{1}{2}-\delta c_{3} \int_{B_{r}(0)} \eta^{2} d x\right)+c_{4}\left|B_{r}(0)\right|\right\} \\
& \leq c_{4}\left|B_{r}(0)\right| .
\end{aligned}
$$

From (10) and (11), we conclude that

$$
M_{0} \int_{\mathbb{R}^{N}}\left|(-\Delta)^{\frac{\alpha}{2}} u_{n}\right|^{2} d x \leq c_{4} N\left|B_{r}(0)\right| .
$$

Note that $E \subset \dot{H}^{\alpha}\left(\mathbb{R}^{N}\right)$ and the embedding $\dot{H}^{\alpha}\left(\mathbb{R}^{N}\right) \hookrightarrow L^{2 *}\left(\mathbb{R}^{N}\right)$ is continuous. Then we have

$$
|u|_{2_{\alpha}^{*}}^{2} \leq \frac{1}{S_{\alpha}} \int_{\mathbb{R}^{N}}\left|(-\Delta)^{\frac{\alpha}{2}} u\right|^{2} d x, \quad \forall u \in E
$$

Recall from (6)

$$
\int_{\mathbb{R}^{N}}\left(\left|(-\Delta)^{\frac{\alpha}{2}} u_{n}\right|^{2}+V(x) u_{n}^{2}\right) d x=N \lambda_{n} \int_{\mathbb{R}^{N}} f\left(u_{n}\right) u_{n} d x .
$$

Using (5) in (13), we obtain

$$
\begin{aligned}
& \int_{\mathbb{R}^{N}}\left(\left|(-\Delta)^{\frac{\alpha}{2}} u_{n}\right|^{2}+V(x) u_{n}^{2}\right) d x \\
& \quad \leq N \int_{\mathbb{R}^{N}}\left(\varepsilon\left|u_{n}\right|^{2}+c_{\varepsilon}\left|u_{n}\right|^{p}\right) d x
\end{aligned}
$$




$$
\begin{aligned}
\leq & N \int_{\mathbb{R}^{N}}\left(\varepsilon \frac{V(x)}{V_{0}}\left|u_{n}\right|^{2}+c_{\varepsilon}\left|u_{n}\right|^{2} \alpha\right) d x \\
\leq & \frac{N \varepsilon}{V_{0}} \int_{\mathbb{R}^{N^{N}}}\left(\left|(-\Delta)^{\frac{\alpha}{2}} u_{n}\right|^{2}+V(x)\left|u_{n}\right|^{2}\right) d x+c_{\varepsilon} N \int_{\mathbb{R}^{N}}\left|u_{n}\right|^{2_{\alpha}^{*}} d x \\
\leq & \frac{N \varepsilon}{V_{0}} \int_{\mathbb{R}^{N}}\left(\left|(-\Delta)^{\frac{\alpha}{2}} u_{n}\right|^{2}+V(x)\left|u_{n}\right|^{2}\right) d x \\
& +c_{\varepsilon} N\left[\frac{1}{S_{\alpha}} \int_{\mathbb{R}^{N}}\left|(-\Delta)^{\frac{\alpha}{2}} u_{n}\right|^{2} d x\right]^{\frac{N}{N-2 \alpha}},
\end{aligned}
$$

which implies that

$$
\begin{aligned}
& \left(1-\frac{N \varepsilon}{V_{0}}\right) \int_{\mathbb{R}^{N}}\left(\left|(-\Delta)^{\frac{\alpha}{2}} u_{n}\right|^{2}+V(x) u_{n}^{2}\right) d x \\
& \leq c_{\varepsilon} N\left[\frac{1}{S_{\alpha}} \int_{\mathbb{R}^{N}}\left|(-\Delta)^{\frac{\alpha}{2}} u_{n}\right|^{2} d x\right]^{\frac{N}{N-2 \alpha}} \\
& \leq c_{\varepsilon} N\left[\frac{1}{S_{\alpha}} \frac{c_{4} N\left|B_{r}(0)\right|}{\alpha}\right]^{\frac{N}{N-2 \alpha}} .
\end{aligned}
$$

It follows that

$$
\left\|u_{n}\right\|_{E}^{2} \leq c_{\varepsilon} N\left[\frac{1}{S_{\alpha}} \frac{c_{4} N\left|B_{r}(0)\right|}{M_{0}}\right]^{\frac{N}{N-2 \alpha}} \frac{V_{0}}{\left(V_{0}-N \varepsilon\right)} .
$$

Then the conclusion holds.

Finally, we are ready to prove our main theorem.

Proof of Theorem 1 Let $u_{n}$ be a critical point for $\varphi_{\lambda_{n}}$ at the level $c_{\lambda_{n}}$. Then from Lemma 7 we may assume that $\left\|u_{n}\right\|_{E} \leq c_{6}$.

Note that $\lambda_{n} \rightarrow 1$, we can show that $\left\{u_{n}\right\}$ is a (PS) sequence of $\varphi$. Indeed, the boundedness of $\left\{u_{n}\right\}$ implies that $\left\{\varphi\left(u_{n}\right)\right\}$ is bounded. Also

$$
\left\langle\varphi^{\prime}\left(u_{n}\right), v\right\rangle=\left\langle\varphi_{\lambda_{n}}^{\prime}\left(u_{n}\right), v\right\rangle+\left(\lambda_{n}-1\right) \int_{\mathbb{R}^{N}} f\left(u_{n}\right) v d x, \quad \forall u \in E .
$$

Hence, $\varphi^{\prime}\left(u_{n}\right) \rightarrow 0$, and consequently $\left\{u_{n}\right\}$ is a bounded (PS) sequence of $\varphi$. By Lemma 5 , $\left\{u_{n}\right\}$ has a convergent subsequence, hence without loss of generality we may assume that $u_{n} \rightarrow u$. Therefore, $\varphi^{\prime}(u)=0$. By virtue of Lemma 4 , we have

$$
\varphi(u)=\lim _{n \rightarrow \infty} \varphi\left(u_{n}\right)=\lim _{n \rightarrow \infty} \varphi_{\lambda_{n}}\left(u_{n}\right) \geq \bar{c}>0,
$$

and $u$ is a positive solution by the condition $H(f)(1)$. The proof is completed.

The authors declare that they have no competing interests. 


\section{Author details}

${ }^{1}$ Department of Applied Mathematics, Harbin Engineering University, Harbin, 150001, P.R. China. ${ }^{2}$ Department of Mathematics, Harbin Institute of Technology, Harbin, 150001, P.R. China.

\section{Acknowledgements}

This work was supported by the National Natural Science Foundation of China (Nos. 11201095, 11201098), the Youth Scholar Backbone Supporting Plan Project of Harbin Engineering University, the Fundamental Research Funds for the Central Universities, Postdoctoral research startup foundation of Heilongjiang (No. LBH-Q14044) and the Science Research Funds for Overseas Returned Chinese Scholars of Heilongjiang Province (No. LC201502).

\section{Received: 14 June 2015 Accepted: 30 November 2015 Published online: 12 December 2015}

\section{References}

1. Autuori, G, Pucci, P: Elliptic problems involving the fractional Laplacian in $\mathbb{R}^{N}$. J. Differ. Equ. 255, 2340-2362 (2013)

2. Chang, XJ: Ground state solutions of asymptotically linear fractional Schrödinger equations. J. Math. Phys. 54, 061504 (2013)

3. Chang, XJ, Wang, ZQ: Ground state of scalar field equations involving a fractional Laplacian with general nonlinearity. Nonlinearity 26, 479-494 (2013)

4. Cheng, M: Bound state for the fractional Schrödinger equation with unbounded potential. J. Math. Phys. 53, 043507 (2012)

5. Felmer, P, Quaas, A, Tan, JG: Positive solutions of nonlinear Schrödinger equation with the fractional Laplacian. Proc. R. Soc. Edinb., Sect. A, Math. 142, 1237-1262 (2012)

6. Secchi, S: Ground state solutions for nonlinear fractional Schrödinger equations in $\mathbb{R}^{N}$. J. Math. Phys. 54, 031501 (2013)

7. Chen, WX, Li, CM, Ou, B: Classification of solutions for an integral equation. Commun. Pure Appl. Math. 59, 330-343 (2006)

8. Nezza, ED, Palatucci, G, Valdinoci, E: Hitchhiker's guide to the fractional Sobolev spaces. Bull. Sci. Math. 136, 521-573 (2012)

9. Ros-Oton, X, Serra, J: The Pohozaev identity for the fractional Laplacian. Arch. Ration. Mech. Anal. 213, 587-628 (2014)

10. Secchi, S: On fractional Schrödinger equations in $\mathbb{R}^{N}$ without the Ambrosetti-Rabinowitz condition (2012). arXiv:1210.0755

11. Struwe, M: Variational Methods: Applications to Nonlinear Partial Differential Equations and Hamiltonian Systems, 4th edn. Ergeb. Math. Grenzgeb. (3), vol. 34. Springer, Berlin (2008)

12. Jeanjean, L: On the existence of bounded Palais-Smale sequences and application to a Landesman-Lazer-type problem set on $\mathbb{R}^{N}$. Proc. R. Soc. Edinb., Sect. A, Math. 129, 787-809 (1999)

\section{Submit your manuscript to a SpringerOpen ${ }^{\circ}$ journal and benefit from:}

- Convenient online submission

Rigorous peer review

- Immediate publication on acceptance

Open access: articles freely available online

- High visibility within the field

- Retaining the copyright to your article 